\title{
An Optically Controlled 3D Cell Culturing System
}

\author{
Kelly S. Ishii, Wenqi Hu, Swapnil A. Namekar, and Aaron T. Ohta \\ Department of Electrical Engineering, University of Hawaii at Manoa, 2540 Dole Street, Holmes Hall 483, \\ Honolulu, HI 96822, USA \\ Correspondence should be addressed to Aaron T. Ohta, aohta@hawaii.edu
}

Received 2 May 2011; Revised 25 August 2011; Accepted 12 September 2011

Academic Editor: Eric Pei Yu Chiou

Copyright $\odot 2011$ Kelly S. Ishii et al. This is an open access article distributed under the Creative Commons Attribution License, which permits unrestricted use, distribution, and reproduction in any medium, provided the original work is properly cited.

\begin{abstract}
A novel 3D cell culture system was developed and tested. The cell culture device consists of a microfluidic chamber on an optically absorbing substrate. Cells are suspended in a thermoresponsive hydrogel solution, and optical patterns are utilized to heat the solution, producing localized hydrogel formation around cells of interest. The hydrogel traps only the desired cells in place while also serving as a biocompatible scaffold for supporting the cultivation of cells in $3 \mathrm{D}$. This is demonstrated with the trapping of MDCK II and HeLa cells. The light intensity from the optically induced hydrogel formation does not significantly affect cell viability.
\end{abstract}

\section{Introduction}

Drug- and cell-based therapies are being explored for many medical conditions [1, 2], including heart disease [3], cancer [4-6], and diabetes [7-9]. However, the process of identifying suitable treatments is long and complicated. The time from drug discovery to Food and Drug Administration approval is usually 10 to 15 years [10], costing from $\$ 800$ million to $\$ 1$ billion [11]. Numerous processes contribute to this long lead time, including the testing of drug compounds, drug effects, and drug safety. These tests are conducted in order of increasing complexity: biochemical assays, cell-based assays, animal models, and then clinical trials. It is possible to simplify this testing procedure with improved cell-based assays that could enable the reduction or elimination of animal testing, reducing the time and cost of developing new drugs.

Cell-based drug assays can be improved by culturing cells in vitro that remain highly representative of their in vivo counterparts, resulting in more realistic testing results, mitigating the risks and costs associated with testing on live subjects, while accelerating the development process. Recent evidence suggests that $3 \mathrm{D}$ cultures are more representative of cellular behavior as compared to 2D cultures [12], as 3D cultures more closely simulate in vivo cellular microenvironments [13]. For example, cancerous cells grown in 3D cultures are more resistant to drugs versus cells in $2 \mathrm{D}$ cultures $[14,15]$.

3D cultures have been developed where cells are seeded on porous biomaterials $[10,15,16]$ or scaffold-like microstructures [17-19]. Integration of these materials in microfluidic systems can facilitate diffusive transfer of nutrients and waste and minimizes the use of materials and reagents, thereby lowering costs and increasing parallelization of culturing and testing $[10,20]$. Thus, appropriately designed microdevices can greatly improve the predictive value of cell-based assays [21-25].

One remaining challenge for microfluidic culture systems is the culturing of specific individual cells. The isolation and culture of specific, individual, and potentially rare cells of interest, such as cancerous cells, can be used to test the effectiveness of drugs, which can lead to the development of treatments that are better tailored to their target. When isolating specific cells, it is desirable to simultaneously select the cells of interest and seed them into a 3D scaffold. This reduces unnecessary handling of the cells, saving time and money, while negating the question of how to recover the selected cells. Minimizing the manipulation required to arrive at the culturing stage also simplifies the equipment necessary to carry out the process.

Here, we demonstrate a microfluidic system that can isolate specific cells from a suspension and seed and culture 
them in $3 \mathrm{D}$ structures. This system creates $3 \mathrm{D}$ hydrogel structures for cell culture using projected light patterns. Specific cells can be captured for homogeneous or heterogeneous $3 \mathrm{D}$ culturing while simultaneously creating the scaffolds necessary to support cell growth in $3 \mathrm{D}$. The 3D hydrogel scaffolds are formed using a thermosensitive polymer instead of photoinitated polymerization $[18,19,21$, $23,24]$, which usually is irreversible. The hydrogel formation is reversible, allowing cultured cells to be easily harvested. In this paper, the details of this optically controlled $3 \mathrm{D}$ culturing system are presented, along with experimental results demonstrating the optically controlled formation of thermosensitive hydrogels, the trapping of micro-objects within hydrogels, the trapping of mammalian cells, and the testing of cell viability.

\section{Optically Controlled 3D Cell Culturing System}

Our cell culture system uses a device with an optically absorbing substrate that converts the optical energy of the light patterns into heat (Figure 1). This heat is used to trigger a sol-gel transition of a thermoresponsive hydrogel containing cells, allowing selective trapping of cells in a $3 \mathrm{D}$ matrix for culturing.

The thermoresponsive hydrogel used is composed of poly( $N$-isopropylacrylamide) (PNIPAAm). This biocompatible polymer dissolves readily in water at room temperature. However, if a PNIPAAm solution is heated above the lower critical solution temperature (LCST) of the PNIPAAm, the polymer undergoes a reversible sol-gel transition, resulting in the creation of a hydrogel [26-28]. As the LCST is approximately $32^{\circ} \mathrm{C}$, PNIPAAm is a popular choice as a thermoresponsive polymer for use with cells and other bioparticles. Thus, PNIPAAm solutions are hydrogels at physiological temperatures but liquids at room temperature.

The optically generated heat in the culture device is sufficient to increase the temperature of the PNIPAAm solution above its LCST, thus inducing a sol-gel transition and forming a 3D hydrogel in the heated region. The dimensions of the hydrogel are determined by the heat distribution generated by the optical patterns, and the height of the microfluidic chamber. The height of the microfluidic chamber in these experiments was maintained at $50 \mu \mathrm{m}$ to ensure sufficient transport of nutrients and waste products into and out of the gels once cells are in culture.

A typical culturing procedure starts by mixing cells at a desired density with a PNIPAAm solution (Figure 2). The solution is introduced into the microfluidic chamber. Optical patterns are then used to form hydrogels around the cells of interest. After trapping the desired cells, the remaining sample is flushed by flowing buffer solution through the device while keeping the gelled region above the LCST. At this point, the optical patterns can be turned off, as long as the device itself is kept above the LCST. The cells can then be cultured. The microfluidic chamber is designed to be removable, providing direct access to the hydrogel, and

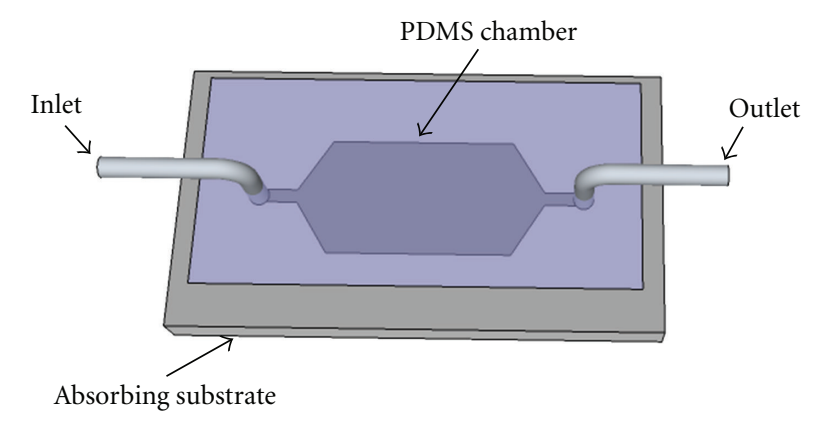

(a)

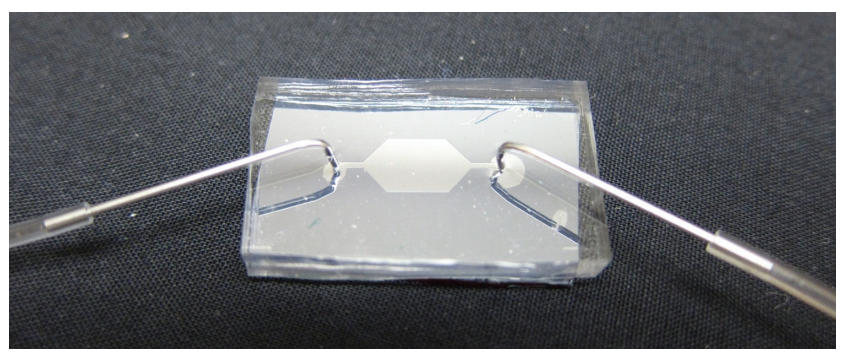

(b)

FIGURE 1: (a) Schematic representation of the 3D cell culture device. A microfluidic chamber is imprinted on polydimethylsiloxane (PDMS) using soft lithography and reversibly bonded to an optically absorbent substrate. Tubing is connected at the inlet and outlet ports. Fluid is introduced at the inlet and is drawn through the device by applying negative pressure at the outlet. (b) A prototype device is shown with fluidic tubing connected at the ports. In this design, the hexagonal chamber is approximately $9 \mathrm{~mm} \times 5 \mathrm{~mm}$; the PDMS piece and accompanying substrate are approximately $30 \mathrm{~mm} \times 15 \mathrm{~mm}$.

making subsequent culture steps more similar to that of a standard 3D culture.

\section{Materials and Methods}

3.1. Thermoresponsive Polymer. A thermoresponsive polymer solution that comprised 10\% (w/v) PNIPAAm (SigmaAldrich, St. Louis, Mo, USA) dissolved in deionized (DI) water or phosphate-buffered saline (PBS) (ATCC, Manassas, $\mathrm{Va}, \mathrm{USA}$ ) was used in the experiments discussed in this paper.

3.2. Cells. Madin Darby Canine Kidney (MDCK II) epithelial cells and HeLa cells were cultivated in a standard 2D culture using Dulbecco's Modified Eagle Medium (DMEM) with $10 \%$ fetal bovine serum (FBS) and $1 \%$ penicillin streptomycin. The cells were incubated at $37^{\circ} \mathrm{C}$ in a humidified 95\% air, 5\% $\mathrm{CO}_{2}$ environment. The cells were harvested using $0.25 \%$ Trypsin/0.53 mM EDTA and mixed into a solution of $10 \%(\mathrm{w} / \mathrm{v})$ PNIPAAm in PBS for the experiments described here. Viability tests were performed using a LIVE/DEAD assay kit (Invitrogen, Carlsbad, Calif, USA) and fluorescent imaging.

3.3. Experimental Setup. The cell culture device is positioned on the stage of an upright microscope, on top of an 


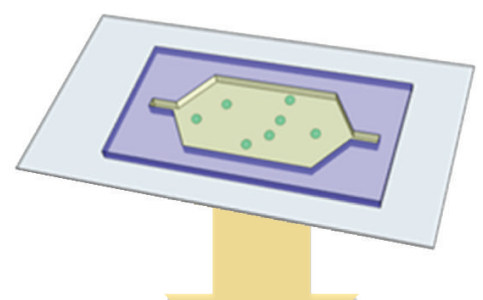

(a) Introduce PNIPAAm solution containing cells

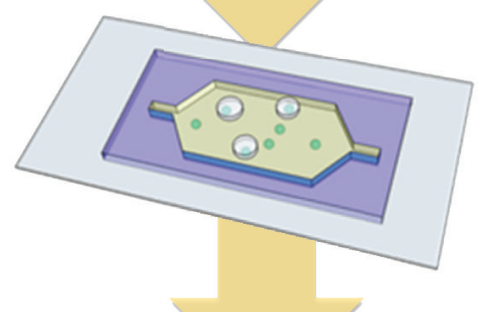

(b) Form gel around desired cells with light
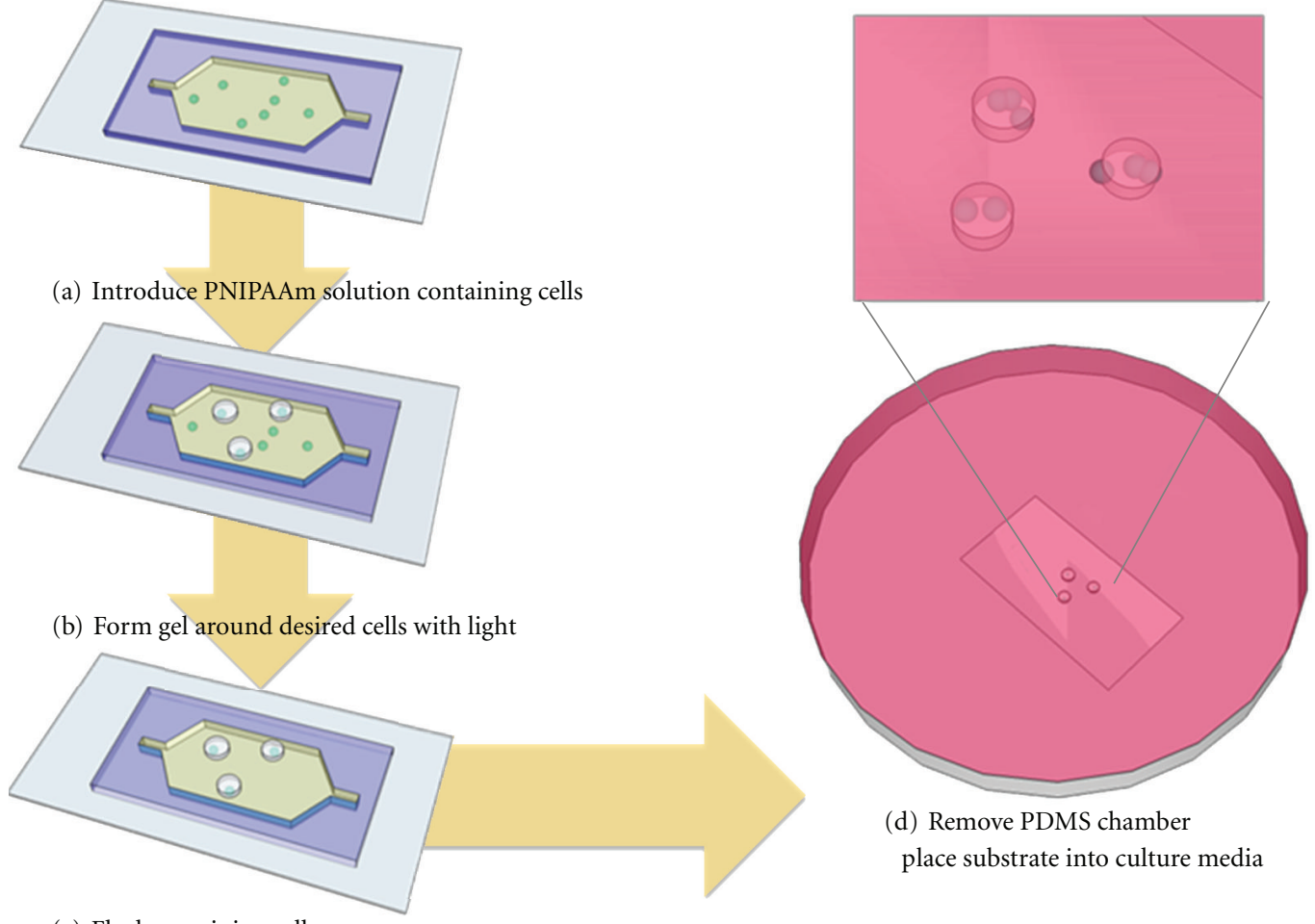

(d) Remove PDMS chamber

place substrate into culture media

(c) Flush remaining cells

FIGURE 2: Optically controlled 3D culturing process. (a) The cell sample in hydrogel solution is introduced to the device chamber. (b) Desired cells are trapped in PNIPAAm hydrogel (cylindrical volume) using localized optical heating. (c) Undesired cells that remain in solution are flushed from the device. (d) While maintaining the hydrogel above the LCST, the PDMS chamber is removed, and the device substrate with the patterned $3 \mathrm{D}$ hydrogel is placed into culture media to allow cell growth.

indium tin oxide (ITO) thin-film heater. The ITO heater is used to warm the device above room temperature, but below the LCST, to facilitate the optically induced gelation. A CCD camera mounted on the microscope provides realtime visualization of the procedure. The thermoresponsive polymer solution containing the cell sample is introduced into the device.

Optical patterns are directed through a hole in the microscope stage to the device substrate. The light patterns are created by focusing the output of a commercial projector (Dell 2400MP) through a 10X objective lens, resulting in an average intensity of $4.07 \mathrm{~W} / \mathrm{cm}^{2}$. An alternate light source that can be used to cause gelation is a $635-\mathrm{nm}, 10-\mathrm{mW}$ diode laser. Irradiating the absorbing substrate results in localized heating within the device.

The 3D culture device consists of a 50- $\mu \mathrm{m}$-high PDMS microfluidic chamber reversibly bonded to an optically absorbent substrate (Figure 1). An inlet port and an outlet port are located at opposite ends of the chamber, and flushing is controlled by negative pressure-driven flow using an external syringe pump.

\section{Experimental Results}

4.1. Device Characterization. The temperature profile in the culture device was simulated using COMSOL Multiphysics (Figure 3). The heat source in this simulation is a $100 \mu \mathrm{m} \times$

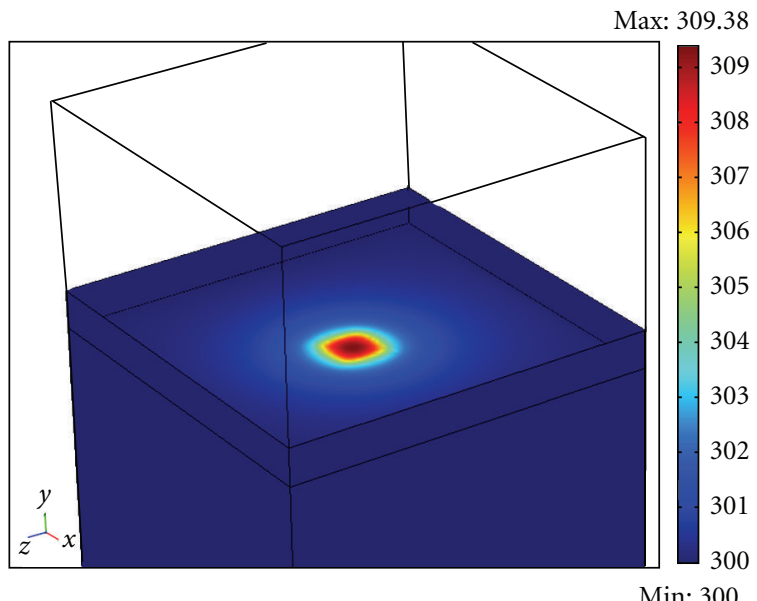

Figure 3: Simulated temperature (degrees Kelvin) in the culture device. In this model, two glass layers sandwich a $100-\mu \mathrm{m}$ thick layer of water. The heat flux is situated at the bottom of the water layer in a $100 \mu \mathrm{m} \times 100 \mu \mathrm{m}$ square area.

$100 \mu \mathrm{m}$, representing a square optical pattern. The heat source is situated at the top surface of the lower glass layer, which corresponds to the location of the optically absorbent layer. This conversion of light to heat is an important parameter in determining the efficacy of the substrate and 
ultimately the resolution of the gel that can be achieved. Thus, various light-absorbent substrates were developed and characterized. The materials used to fabricate the substrates were chosen on the basis that they were readily available as well as biocompatible. The primary substrate used consists of a $1.1-\mathrm{mm}$-thick glass slide coated with a 200 -nm-thick layer of ITO and a 1- $\mu \mathrm{m}$-thick layer of amorphous silicon (a-Si). The a-Si layer absorbs near-infrared, visible, and UV light, converting it to heat. A simpler substrate was also tested, consisting of a $150-\mu \mathrm{m}$-thick glass coverslip backed with a layer of black vinyl tape (3M Corp., St. Paul, Minn, USA). Additionally, absorbing polymeric substrates were created by mixing dark particles such as black mineral pigment and carbon black with PDMS.

In order to precisely select single, individual cells for culture, the hydrogel resolution needs to be optimized. While it is desirable to obtain the smallest hydrogel area necessary to fully encompass the cell, the area of hydrogel should also remain relatively constant over the short period of time between trapping and flushing steps. If the heated region of the substrate continues to spread from the point where the light pattern is aimed, the hydrogel region will continue to expand, potentially trapping additional, undesired cells in the immediate area. For this purpose, the various substrates were compared by measuring the area of the hydrogel while exposing the device to the smallest circular light pattern capable of producing gel within the first 30 seconds of exposure. First, the minimum optical pattern dimensions that produced gelling were determined for each substrate. The time point at which hydrogel initially appeared varied across the substrates. The area of the hydrogel was measured over an exposure time of $60 \mathrm{~s}$ to include the effects of continued hydrogel formation with time (Figure 4). The diameters of the circular optical patterns used for the different substrates were mineral pigment $(450 \mu \mathrm{m})$, electrical tape on a glass coverslip $(550 \mu \mathrm{m})$, carbon black $(420 \mu \mathrm{m})$ and a-Si $(580 \mu \mathrm{m})$. The a-Si substrate was used in the subsequent experiments presented here, as it made the microscope images within the microfluidic chamber clearer. The absorptive nature of the surface of other substrates hinders reflective microscopy, which was used in the experiments here. The ability to visualize the cells is crucial in differentiating anomalous cells from the bulk of a suspension, so the superior imaging qualities of the a-Si substrate made it easier to work with than the other substrates.

The resolution of this technique can be improved by replacing the computer projector with a laser operating in the near-infrared, visible, or UV wavelength range. Hydrogel formation using the computer projector light source is limited by the intensity of the light from the lamp in the projector. The relatively low intensity requires larger illuminated areas to create sufficient heat to trigger the PNIPAAm gelation. Thus, the resolution of hydrogel formation was examined further using the a-Si substrate and a 635$\mathrm{nm}, 10-\mathrm{mW}$ diode laser as the optical source. The laser was focused down to a spot of approximately $108 \mu \mathrm{m}^{2}$. The substrate was irradiated for $30 \mathrm{~s}$ at various laser powers, and the resulting hydrogel area was measured (Figure 5).

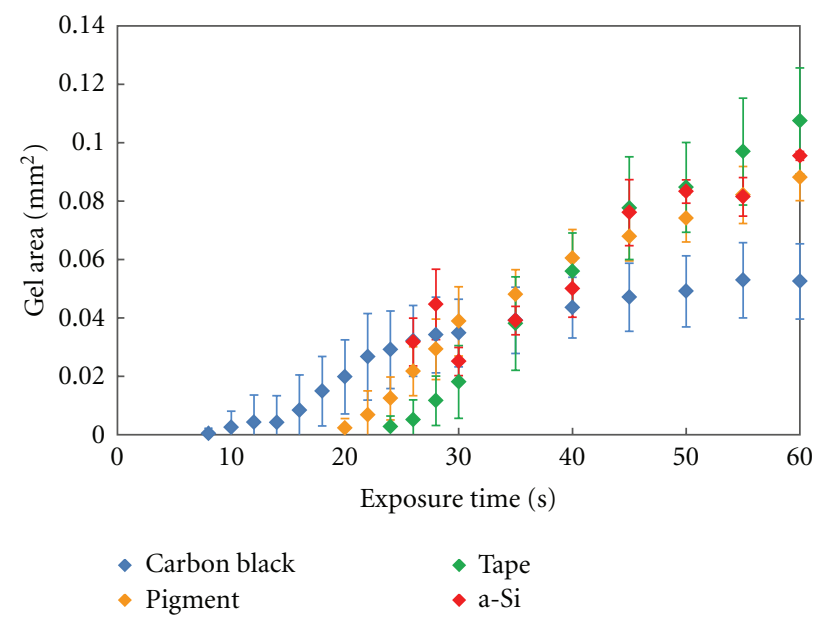

Figure 4: Hydrogel area versus time for various optically absorbent substrates. The optically smallest pattern that effectively produced gel was determined for each substrate, using the computer projector light source. The graph shows the average hydrogel area measured across five trials and the standard deviation for each. A two-minute delay between subsequent trials allowed sufficient time for the device to return to room temperature.

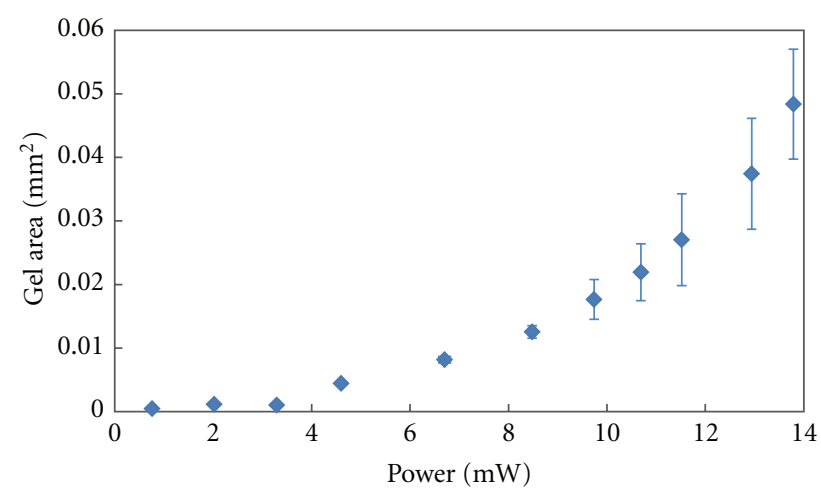

FIGURE 5: Hydrogel area as a function of power, using a 635-nm diode laser as the optical source. The area of the laser spot was approximately $108 \mu \mathrm{m}^{2}$. The substrate was irradiated for a period of 30 seconds to generate each data point. The graph shows the average hydrogel area measured across five trials and the standard deviation of the measurements.

The smallest resultant gel area was $182 \mu \mathrm{m}^{2}$. To remove the effect of accumulated substrate heating, measurement at each power level was performed at a different location in the microfluidic chamber. The gels formed with the laser have a smaller area than those produced using the computer projector. However, since the laser is focused into a single point, it does not provide as much flexibility as the projection of computer-drawn patterns. Thus, the trapping experiments utilized the computer projector setup although more flexibility would be possible by using holographic imaging or spatial light modulators, similar to those used in holographic optical tweezers [29].

4.2. Microbead Trapping. The capability of trapping specific objects in 3D hydrogels was demonstrated using polystyrene 


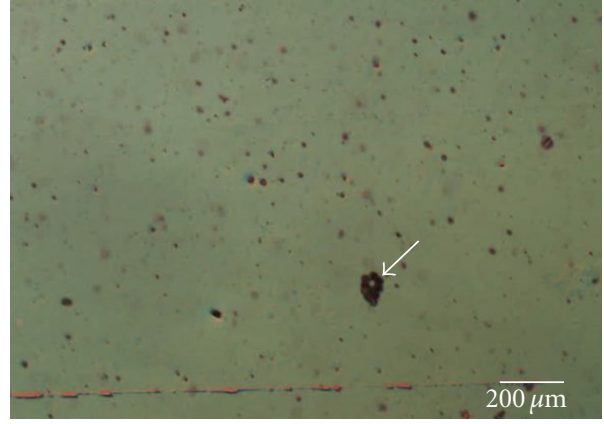

(a)

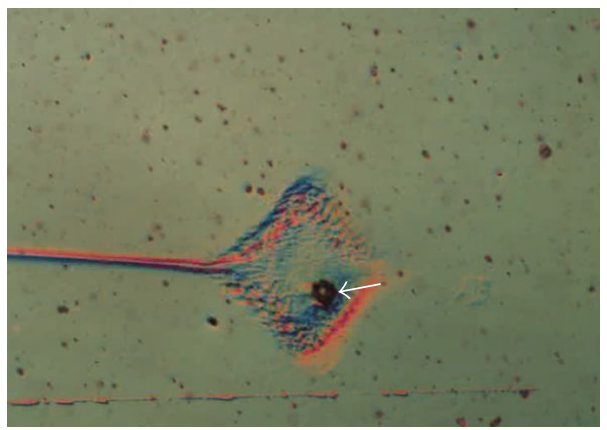

(c)

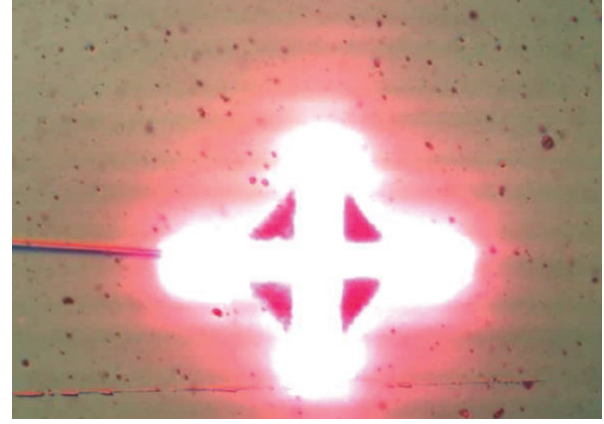

(b)

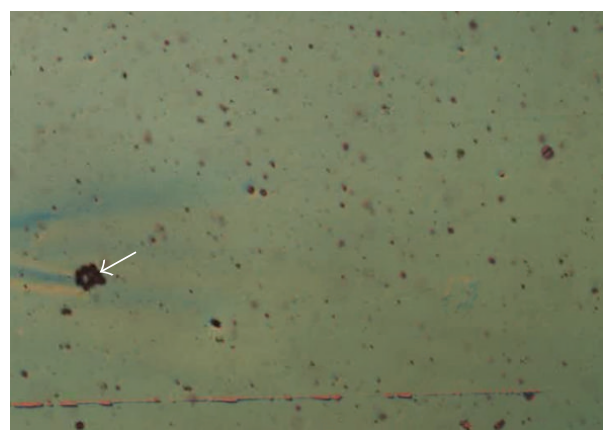

(d)

FIGURE 6: Trapping of 20- $\mu \mathrm{m}$-diameter polystyrene microbeads in an optically patterned hydrogel. The scale bar is $200 \mu \mathrm{m}$. (a) A PNIPAAm solution containing 10- and 20- $\mu \mathrm{m}$-diameter microbeads was introduced into the microfluidic chamber. The solution is flowing from the right to the left at a linear flow rate of $100 \mu \mathrm{m} / \mathrm{s}$. A clump of beads was targeted for trapping, as indicated by the arrow. (b) An optical pattern is used to heat the substrate, causing a hydrogel to form in the illuminated area. (c) The hydrogel with the optical pattern removed, showing the trapped target particles. (d) Substrate cooling in the absence of optical heating reverses the trap, and the beads begin to flow away.

beads, which also served as model particles for cells. A group of $20-\mu \mathrm{m}$-diameter polystyrene microbeads was selectively trapped from a solution containing 10 -and $20-\mu \mathrm{m}$ beads using optically induced hydrogel formation (Figure 6). Here, polystyrene particles were added to the PNIPAAm solution and flowed through the microfluidic chamber at a linear flow rate of $100 \mu \mathrm{m} / \mathrm{s}$. A target was identified, consisting of a clump of beads (Figure 6(a)). An appropriate light pattern was activated to trigger hydrogel formation, trapping the target group of beads (Figures 6(b) and 6(c)). Other beads in the solution were diverted around the hydrogel formation. Switching off the light pattern allows the substrate temperature to decrease, reducing the amount of hydrogel. As the gel reverts back to its sol state, the trapped beads are released, and begin to wash away with the fluid flow (Figure 6(d)).

4.3. Mammalian Cells. The optically controlled culturing system was also tested with mammalian cells. Single- and multiple-cell trapping as well as parallel trapping were demonstrated with MDCK II cells. The cells were added to PNIPAAm/PBS solution, resulting in a cell density of approximately $1 \times 10^{5}$ cells $/ \mathrm{mL}$ and $10 \%(\mathrm{w} / \mathrm{v})$ concentration of PNIPAAm. The MDCK II cells were selectively trapped using optically induced hydrogel formation (Figure 7). Initially, the cell solution was introduced into the microfluidic chamber, and target cells were selected (Figures 7(a) and 7(d)). Under no-flow conditions, appropriate light patterns were activated to trigger hydrogel formation in the immediate surrounding area, trapping specific cells. Single cells can be trapped, (Figures $7(\mathrm{a})-7(\mathrm{c})$ ) or multiple cells (Figures $7(\mathrm{~d})-7(\mathrm{f})$ ). Cells can be trapped in parallel by using multiple light patterns (Figure 7(e)). Cells that are not trapped are flushed by flowing PNIPAAm/PBS or buffer solution until the free cells are flushed away, leaving only the cells within the optically patterned gels (Figures 7(c) and 7(f)). At this point, the light patterns can be turned off as long as the device temperature is maintained above $32^{\circ} \mathrm{C}$.

Cell viability tests on MDCK II and HeLa cells were performed immediately after the trapping procedure using a fluorescent LIVE/DEAD assay. The cells were suspended in $10 \%(\mathrm{w} / \mathrm{v})$ PNIPAAm in PBS. The cells were irradiated with a circular light pattern $200 \mu \mathrm{m}$ in diameter for approximately 20 minutes while trapping and flushing took place (Figures $8(\mathrm{a})$ and $9(\mathrm{a}))$. Buffer solution was used to remove the cells not trapped in the gel from the device, and then, PBS solution containing the viability assay components was flushed in. As soon as flow was stopped, the light pattern was removed, and the temperature of the ITO heater was raised above the LCST to compensate for the loss of heat. The cells were incubated for 25-30 minutes, as the stain was allowed to permeate the hydrogel. Cell viability of a MDCK II sample was approximately $100 \%$, with no dead cells visibly fluorescing (Figure 8(b)). In the experiment with 


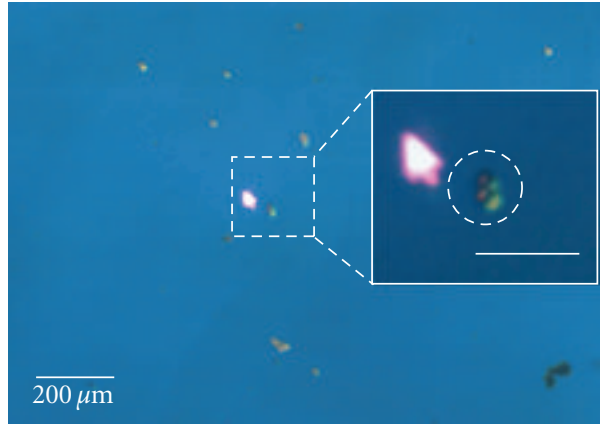

(a)

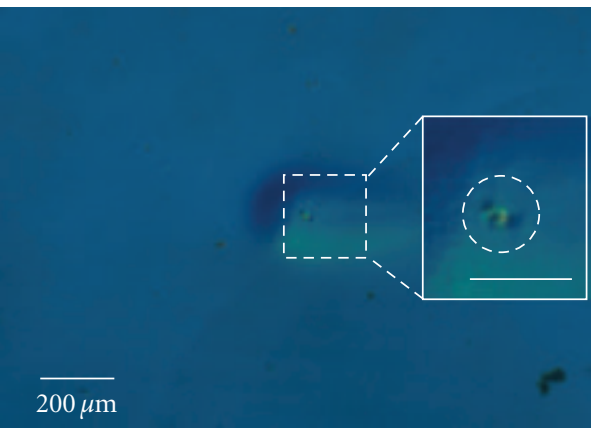

(c)

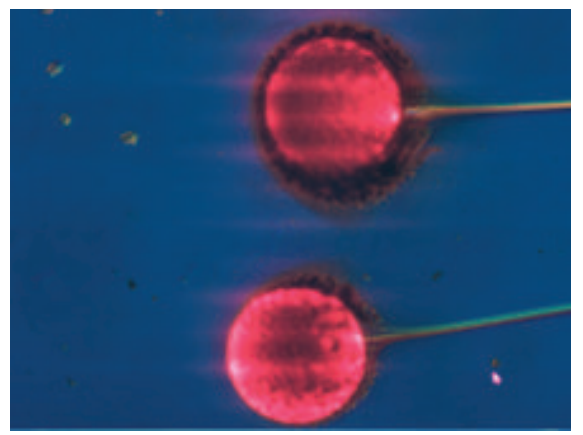

(e)

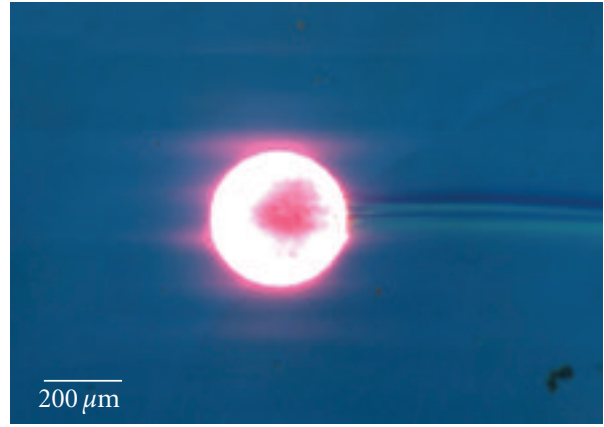

(b)

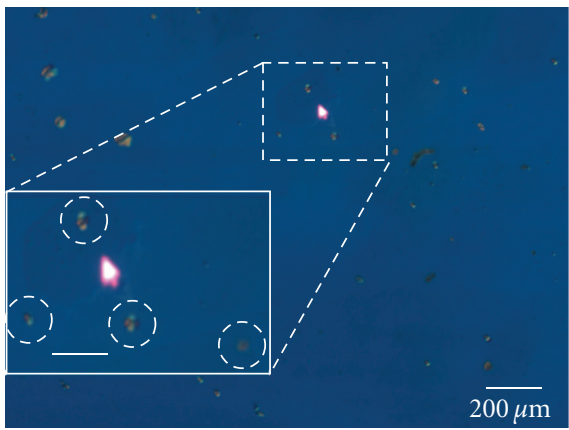

(d)

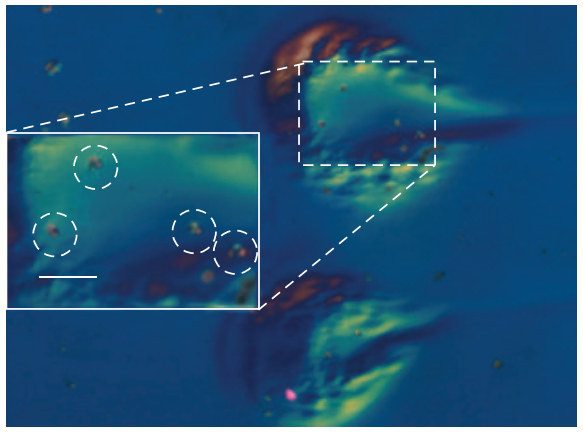

(f)

Figure 7: Gelation of hydrogels using optical control, demonstrating the trapping of MDCK II cells in 3D hydrogels. (a) Initial cell distribution. The two target cells are circled in the inset. Other cells will be flushed away. The inset scale bar is $100 \mu \mathrm{m}$. (b) A light pattern (bright circle) is used to trigger hydrogel formation (dark circular area), trapping the target cells. Cells not trapped in the gel are flushed away. (c) The two target cells remain in the 3D hydrogel. (d) Initial cell distribution when using parallel optical patterns to trap MDCK II cells in 3D hydrogels. Four target cells are circled in the inset. Other cells will be flushed away, except those trapped by the second optical pattern. The inset scale bar is $100 \mu \mathrm{m}$. (e) Light patterns (bright circles) are used to trigger hydrogel formation (dark circular areas), trapping the target cells while free cells are flushed away. (f) The four target cells remain in the 3D hydrogel.

HeLa cells, over $80 \%$ of the cells entrapped in the optically patterned hydrogel fluoresced green, indicating that the cells survive the process (Figure 9(b)). Some cells at the border of the hydrogel exhibited the red fluorescence indicative of cell death (Figure 9(c)). It was expected that the trapping procedure should have an insignificant effect on viability, as PNIPAAm is biocompatible [27, 28]. Furthermore, the cells are exposed to an optical intensity of only $0.17 \mathrm{~W} / \mathrm{cm}^{2}$, as most of the incident light is absorbed by the substrate.
This intensity should not directly cause increased DNA fragmentation [30]. These properties make our technique safe for the culturing of specific cells in 3D.

\section{Conclusion}

In this work, a cell culture system was demonstrated with the ability to trap cells of interest in reversible 3D scaffolds composed of a thermoresponsive hydrogel, PNIPAAm. 


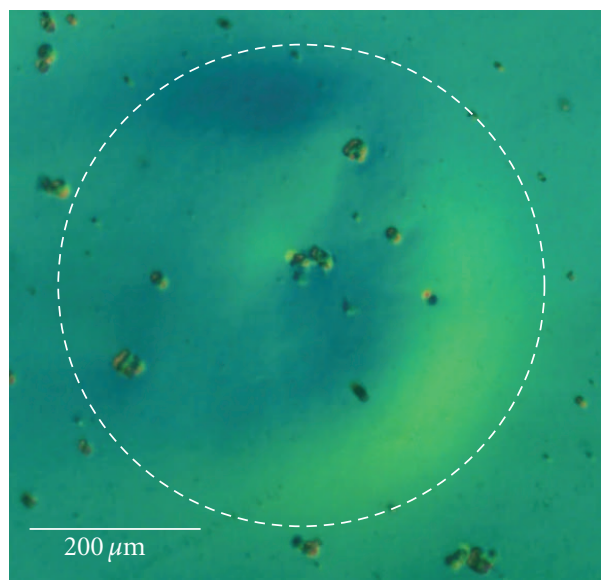

(a)

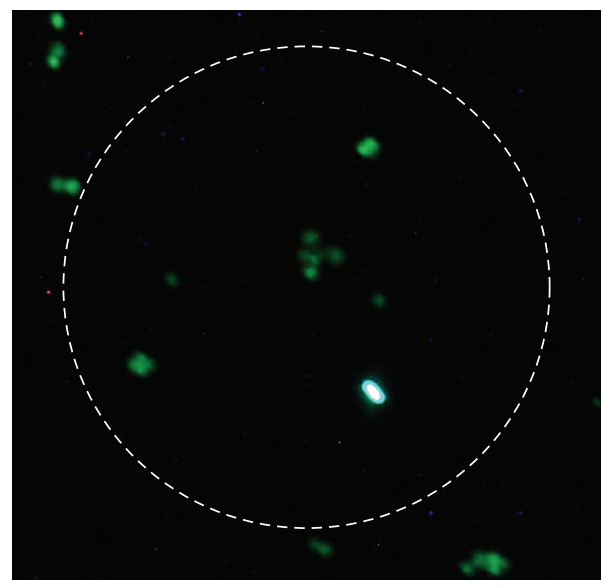

(b)

FIGURE 8: Cell viability testing using LIVE/DEAD assay on trapped MDCK II cells. (a) Initial distribution of MDCK II cells after 50 minutes in an optically patterned hydrogel. The starting cell density was approximately $1 \times 10^{5} \mathrm{cells} / \mathrm{mL}$ in solution. The dashed circle encloses the area that was gelled. The circular objects that are visible are the cells. (b) Fluorescent image of the same cells from (a), demonstrating approximately $100 \%$ viability. Cells lying outside the gel were not taken into account. Live cells fluoresce green, while dead cells fluoresce red. Fluorescent cells appear as bright spots in these images.

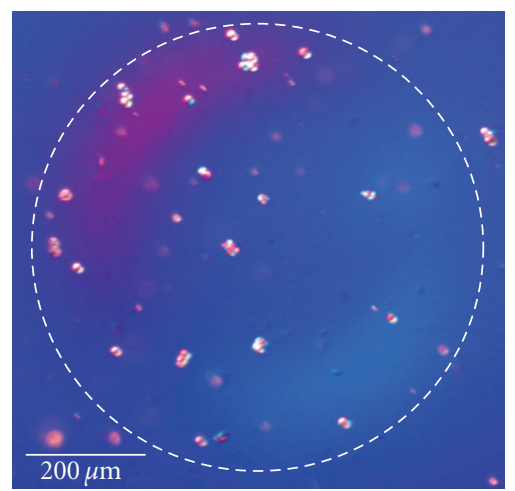

(a)

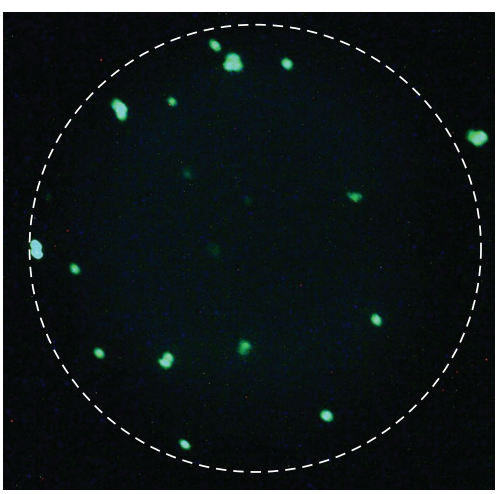

(b)

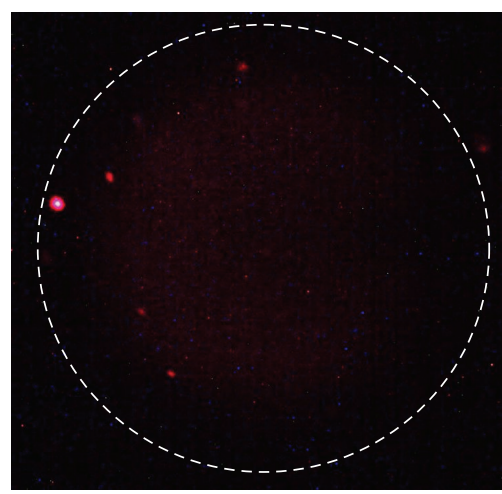

(c)

FIgURE 9: Cell viability testing using LIVE/DEAD assay on trapped HeLa cells. (a) Initial distribution of HeLa cells after 45 minutes in an optically patterned hydrogel. The starting cell density was approximately $1 \times 10^{6}$ cells $/ \mathrm{mL}$ in solution. (b-c) Fluorescent images of the same cells from (a), demonstrating approximately $81 \%$ viability. Live cells fluoresce green (b), while dead cells fluoresce red (c). Fluorescent cells appear as bright spots in these images.

The culture system utilizes a simple microfluidic device that acts as a repository for the hydrogel solution containing the cell sample while the trapping procedure is performed. Optically generated heating is used to induce localized gelation of cells within the device, enabling the trapping of specific cells. Two possible methods of generating localized heating at the cellular level were shown: using a modified commercial projector as a source of light and using a diode laser. The projector offers a higher degree of flexibility for pattern generation, whereas the higher intensity of the laser provides a stronger heat source and can thus create gels with smaller areas. The intensity of either source is not at a level where it is expected to damage the irradiated cells. To test the trapping capabilities of the culture system, experiments involving microbeads as well as MDCK II and HeLa cells have been performed. With MDCK II cells, single- and multiplecell trapping was achieved, using a single light pattern or multiple simultaneous patterns. Preliminary viability tests show that over $80 \%$ cells survive the trapping procedure. Future work includes performing further viability tests at later time points during the cell cultivation process.

\section{Acknowledgments}

The authors would like to thank Yujia Li and Dr. Hongwei Li for providing the MDCK II cells and Dr. Ava Dykes and Dr. Shiv Sharma for the HeLa cells. This project is supported by the National Science Foundation, Grant no. EEC0926632. The project described was also supported by Grant no. U54RR022762 from the National Center for Research Resources (NCRR), a component of the National Institutes of Health (NIH), and its contents are solely the responsibility of the authors and do not necessarily represent the official view of NCRR or NIH. 


\section{References}

[1] A. Dove, "Cell-based therapies go live," Nature Biotechnology, vol. 20, no. 4, pp. 339-343, 2002.

[2] K. H. Wilan, C. T. Scott, and S. Herrera, "Chasing a cellular fountain of youth," Nature Biotechnology, vol. 23, no. 7, pp. 807-815, 2005.

[3] V. F. M. Segers and R. T. Lee, "Stem-cell therapy for cardiac disease," Nature, vol. 451, no. 7181, pp. 937-942, 2008.

[4] W. Xu, L. Liu, and I. G. Charles, "Microencapsulated iNOSexpressing cells cause tumor suppression in mice," The FASEB Journal, vol. 16, no. 2, pp. 213-215, 2002.

[5] M. E. Dudley and S. A. Rosenberg, "Adoptive-cell-transfer therapy for the treatment of patients with cancer," Nature Reviews Cancer, vol. 3, no. 9, pp. 666-675, 2003.

[6] L. Gattinoni, D. J. Powell, S. A. Rosenberg, and N. P. Restifo, "Adoptive immunotherapy for cancer: building on success," Nature Reviews Immunology, vol. 6, no. 5, pp. 383-393, 2006.

[7] Y. Sun, X. Ma, D. Zhou, I. Vacek, and A. M. Sun, "Normalization of diabetes in spontaneously diabetic cynomologus monkeys by xenografts of microencapsulated porcine islets without immunosuppression," Journal of Clinical Investigation, vol. 98, no. 6, pp. 1417-1422, 1996.

[8] T. Sapir, K. Shternhall, I. Meivar-Levy et al., "Cell-replacement therapy for diabetes: generating functional insulin-producing tissue from adult human liver cells," Proceedings of the National Academy of Sciences of the United States of America, vol. 102, no. 22, pp. 7964-7969, 2005.

[9] J. J. Meier, A. Bhushan, and P. C. Butler, "The potential for stem cell therapy in diabetes," Pediatric Research, vol. 59, no. 4, pp. 65R-73R, 2006.

[10] M. H. Wu, S. B. Huang, and G. B. Lee, "Microfluidic cell culture systems for drug research," Lab on a Chip, vol. 10, no. 8, pp. 939-956, 2010.

[11] Pharmaceutical Research and Manufacturers of America, Pharmaceutical Industry Profile2011, PhRMA, Washington, DC, USA, 2011, http://www.phrma.org/.

[12] U. Marx and V. Sandig, Drug Testing In Vitro: Breakthroughs and Trends in Cell Culture Technology, Wiley-VCH, Weinheim, Germany, 2007.

[13] J. El-Ali, P. K. Sorger, and K. F. Jensen, "Cells on chips," Nature, vol. 442, no. 7101, pp. 403-411, 2006.

[14] Y. S. Torisawa, H. Shiku, T. Yasukawa, M. Nishizawa, and T. Matsue, "Multi-channel 3-D cell culture device integrated on a silicon chip for anticancer drug sensitivity test," Biomaterials, vol. 26, no. 14, pp. 2165-2172, 2005.

[15] D. W. Hutmacher, "Biomaterials offer cancer research the third dimension," Nature Materials, vol. 9, no. 2, pp. 90-93, 2010.

[16] B. A. Justice, N. A. Badr, and R. A. Felder, "3D cell culture opens new dimensions in cell-based assays," Drug Discovery Today, vol. 14, no. 1-2, pp. 102-107, 2009.

[17] R. C. Dutta and A. K. Dutta, "Cell-interactive 3D-scaffold; advances and applications," Biotechnology Advances, vol. 27, no. 4, pp. 334-339, 2009.

[18] L. H. Han, G. Mapili, S. Chen, and K. Roy, "Projection microfabrication of three-dimensional scaffolds for tissue engineering," Journal of Manufacturing Science and Engineering, vol. 130, no. 2, article 021005, 4 pages, 2008.

[19] V. Chan, P. Zorlutuna, J. H. Jeong, H. Kong, and R. Bashir, "Three-dimensional photopatterning of hydrogels using stereolithography for long-term cell encapsulation," Lab on a Chip, vol. 10, no. 16, pp. 2062-2070, 2010.
[20] P. S. Dittrich and A. Manz, "Lab-on-a-chip: microfluidics in drug discovery," Nature Reviews Drug Discovery, vol. 5, no. 3, pp. 210-218, 2006.

[21] V. L. Tsang and S. N. Bhatia, "Three-dimensional tissue fabrication," Advanced Drug Delivery Reviews, vol. 56, no. 11, pp. 1635-1647, 2004.

[22] M. J. Powers, K. Domansky, M. R. Kaazempur-Mofrad et al., "A microfabricated array bioreactor for perfused 3D liver culture," Biotechnology and Bioengineering, vol. 78, no. 3, pp. 257-269, 2002.

[23] S. A. Lee, S. E. Chung, W. Park, S. H. Lee, and S. Kwon, "Threedimensional fabrication of heterogeneous microstructures using soft membrane deformation and optofluidic maskless lithography," Lab on a Chip, vol. 9, no. 12, pp. 1670-1675, 2009.

[24] D. R. Albrecht, G. H. Underhill, T. B. Wassermann, R. L. Sah, and S. N. Bhatia, "Probing the role of multicellular organization in three-dimensional microenvironments," Nature Methods, vol. 3, no. 5, pp. 369-375, 2006.

[25] T. Braschler, R. Johann, M. Heule, L. Metref, and P. Renaud, "Gentle cell trapping and release on a microfluidic chip by in situ alginate hydrogel formation," Lab on a Chip, vol. 5, no. 5, pp. 553-559, 2005.

[26] Y. H. Bae, T. Okano, and S. Wan Kim, “Temperature dependence of swelling of crosslinked poly $(N, N$ '-alkyl substituted acrylamides) in water," Journal of Polymer Science B, vol. 28, no. 6, pp. 923-936, 1990.

[27] R. A. Stile, W. R. Burghardt, and K. E. Healy, "Synthesis and characterization of injectable poly ( $N$-isopropylacrylamide)based hydrogels that support tissue formation in vitro," Macromolecules, vol. 32, no. 22, pp. 7370-7379, 1999.

[28] J. F. Pollock and K. E. Healy, "Mechanical and swelling characterization of poly $(N$-isopropyl acrylamide -co- methoxy poly(ethylene glycol) methacrylate) sol-gels," Acta Biomaterialia, vol. 6, no. 4, pp. 1307-1318, 2010.

[29] D. G. Grier, “A revolution in optical manipulation,” Nature, vol. 424, no. 6950, pp. 810-816, 2003.

[30] S. K. Mohanty, A. Rapp, S. Monajembashi, P. K. Gupta, and K. O. Greulich, "Comet assay measurements of DNA damage in cells by laser microbeams and trapping beams with wavelengths spanning a range of $308 \mathrm{~nm}$ to $1064 \mathrm{~nm}$," Radiation Research, vol. 157, no. 4, pp. 378-385, 2002. 

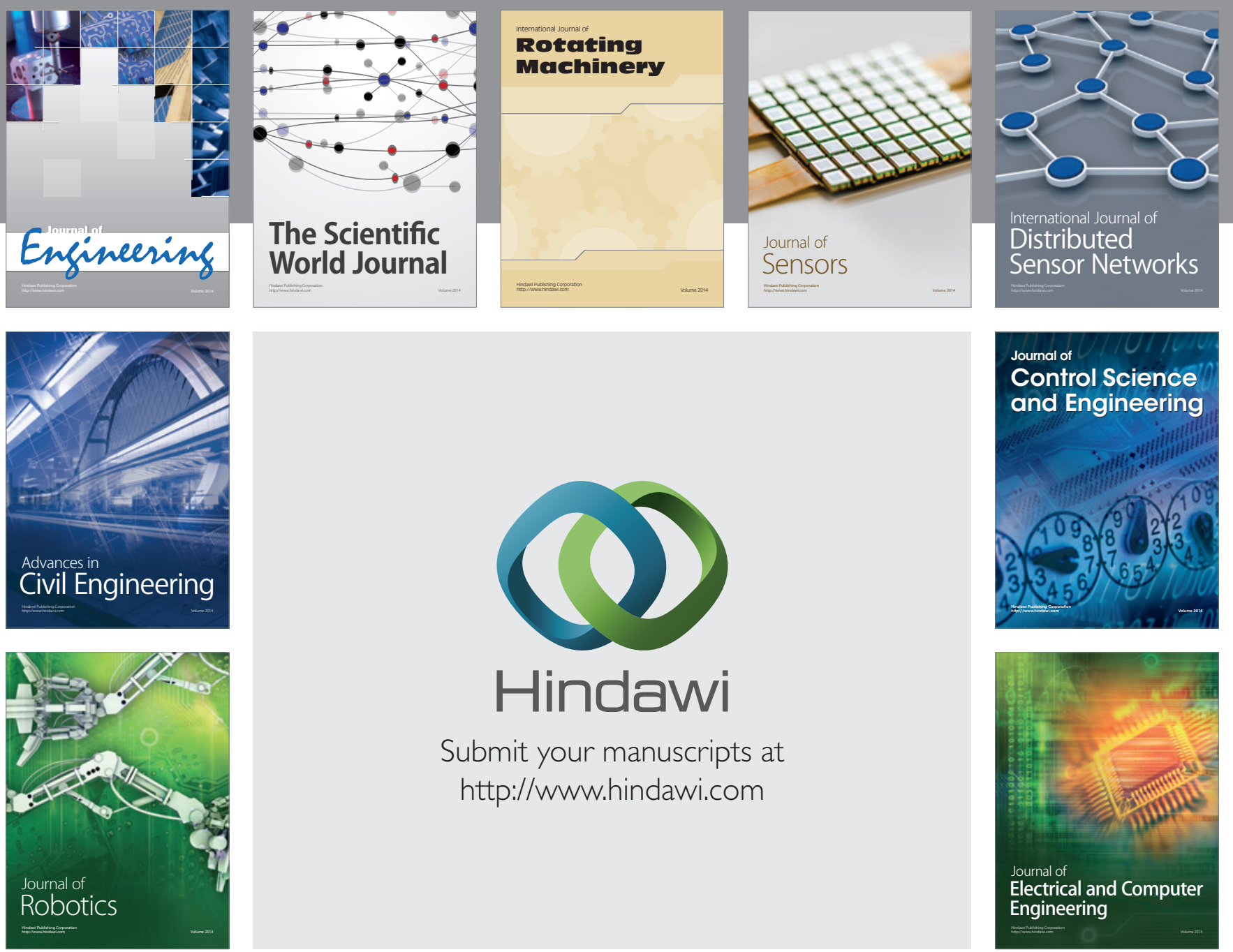

Submit your manuscripts at

http://www.hindawi.com
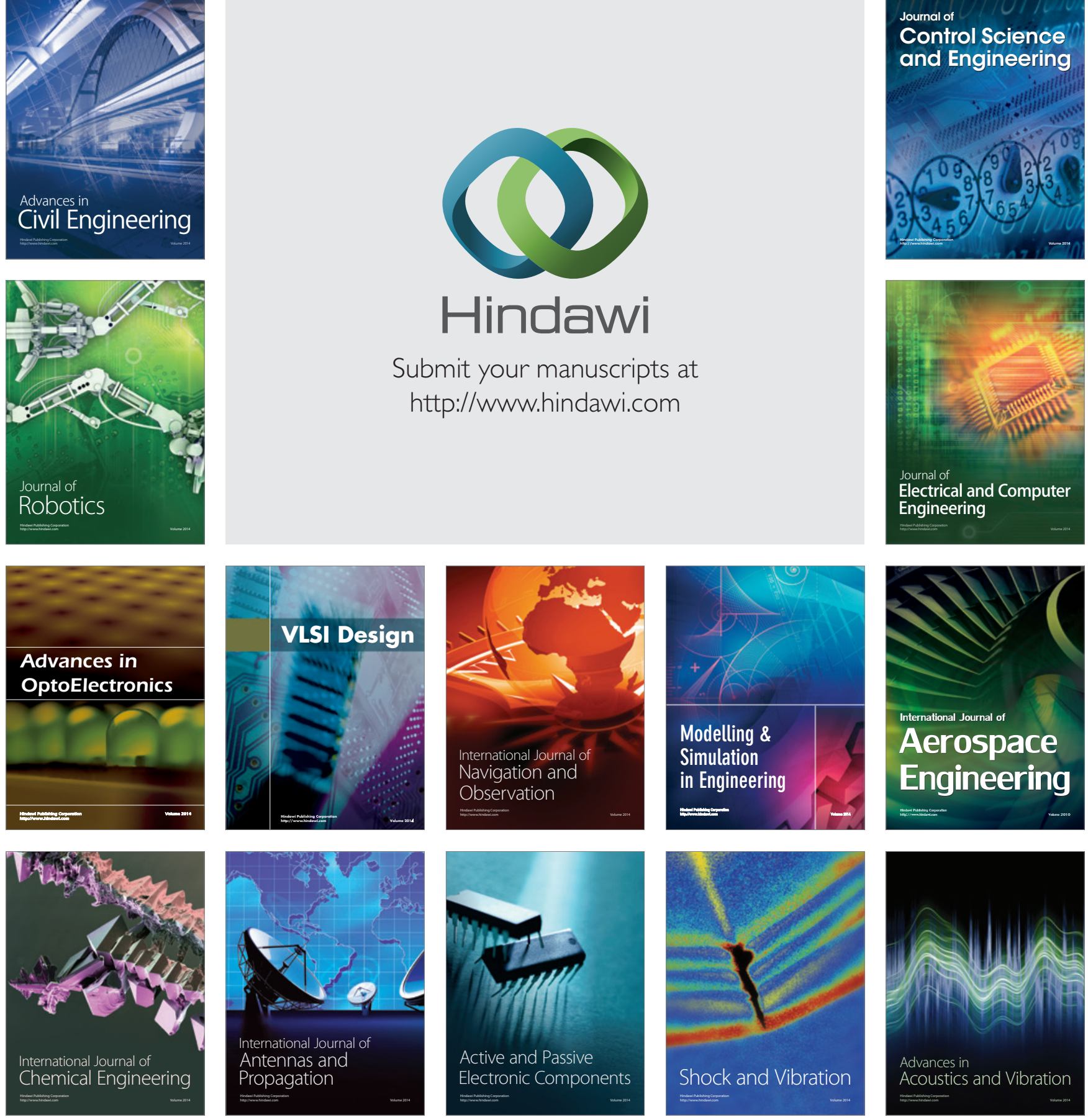